Tropical Journal of Pharmaceutical Research June 2015; 14 (6): 1081-1088

ISSN: $1596-5996$ (print); 1596-9827 (electronic)

(C) Pharmacotherapy Group, Faculty of Pharmacy, University of Benin, Benin City, 300001 Nigeria.

All rights reserved.

Available online at http://www.tjpr.org

Original Research Article

http://dx.doi.org/10.4314/tjpr.v14i6.21

\title{
Synthesis of N'-Substituted-2-(5-(4-Chlorophenyl)-1,3,4- oxadiazol-2-ylthio)acetohydrazide Derivatives as Suitable Antibacterial Agents
}

\author{
S Rasool ${ }^{1}$, Aziz-ur-Rehman ${ }^{1 *}$, MA Abbasi ${ }^{1}$, S Gul $^{1}$, MN Akhtar ${ }^{2}$, I Ahmad ${ }^{3}$ and S \\ Afzal $^{3}$ \\ ${ }^{1}$ Department of Chemistry, Government College University, Lahore-54000, Pakistan, ${ }^{2}$ Faculty of Industrial Sciences \& \\ Technology (FIST), University Malaysia Pahang (UMP), Lebuhraya Tun Razak 26300 Gambang, Kuantan, Malaysia, \\ ${ }^{3}$ Department of Pharmacy, The Islamia University of Bahawalpur, Bahawalpur-63100, Pakistan
}

*For correspondence: Email: azizryk@yahoo.com; Tel: (+92)-42-111000010 ext 449

Received: 24 May 2014

Revised accepted: 12 April 2015

\begin{abstract}
Purpose: To evaluate antibacterial activity of a series of molecules bearing 1,3,4-oxadiazole and azomethine moieties.

Methods: The 4-chlorobenzoic acid (1) was precursor to N'-substituted-2-(5-(4-chlorophenyl)-1,3,4oxadiazol-2-ylthio)acetohydrazide, 8a-p, through a multistep synthesis of corresponding ester, 2, hydrazide, $\mathbf{3}$ and 1,3,4-oxadiazole, 4. The molecule, 4, was subjected to electrophilic substitution by ethyl-2-bromoacetate to yield 5 which was stepped to 2-(5-(4-chlorophenyl)-1,3,4-oxadiazol-2ylthio)acetohydrazide (6). The target molecules, 8a-p, were synthesized by nucleophilic addition of 6 to arylaldehydes, 7a-p. The proposed structures of all the synthesized molecules were elucidated by Infra Red (IR), Proton Nuclear Magnetic Resonance $\left({ }^{1} H-N M R\right)$ and Electron Impact Mass Spectrometry (EIMS) spectral data. Antibacterial activity was evaluated by the principle that microbial growth is in a log phase of growth and so results in increased absorbance of broth medium which is observed.

Results: The molecule, $\mathbf{8 b}$, was active against $S$. aureus and $\mathbf{8 c}$ against $S$. typhi only. The molecule, $\mathbf{8 p}$, was the most active against S. typhi with minimum inhibitory concentration (MIC) value of $10.04 \pm$ $1.25 \mu \mathrm{M}$ while $8 \mathrm{e}$ was active against $E$. coli with MIC of $9.45 \pm 1.00 \mu \mathrm{M}$, both relative to the reference standard, ciprofloxacin, which displayed MIC of $9.13 \pm 2.00$ and $8.90 \pm 1.65 \mu \mathrm{M}$, respectively.

Conclusion: Most of the synthesized molecules exhibit $50 \%$ antibacterial activity relative to the reference. Molecules $\mathbf{8 b}$ and $\mathbf{8 c}$ are the least active compounds.
\end{abstract}

Keywords: 1,3,4-Oxadiazole, 4-Chlorobenzoic acid, Antibacterial activity, Azomethine

Tropical Journal of Pharmaceutical Research is indexed by Science Citation Index (SciSearch), Scopus, International Pharmaceutical Abstract, Chemical Abstracts, Embase, Index Copernicus, EBSCO, African Index Medicus, JournalSeek, Journal Citation Reports/Science Edition, Directory of Open Access Journals (DOAJ), African Journal Online, Bioline International, Open-J-Gate and Pharmacy Abstracts

\section{INTRODUCTION}

Bacteria have developed resistance to several antibiotics over time. Scientists have continued for several years to discover new potent molecules with remarkable activities [1]. Heterocyclic compounds have often been synthesized because of their broad spectrum of biological activities [2]. The 2,5-substituted-1,3,4- oxadiazole derivatives, which have often been synthesized in the last decade, exhibit a number of biological activities [3-7] . In continuation of a previous synthetic work using 1,3,4-oxadiazole derivatives [8,9], N-substituted derivatives of 2(5-(4-chlorophenyl)-1,3,4-oxadiazol-2-

ylthio)acetohydrazide have been synthesized in this study to determine their antibacterial potential. 


\section{EXPERIMENTAL}

\section{General}

Melting points of the synthesized compounds were carried out in a Griffin-George apparatus with open capillary tube and were uncorrected; I.R. spectral data by $\mathrm{KBr}$ pellet on a Jasco-320-A spectrophotometer; ${ }^{1} \mathrm{H}-\mathrm{NMR}$ spectral data by Bruker spectrometer in dimethylsulfoxide (DMSO) at $600 \mathrm{MHz}$; and EIMS spectral data by JMS-HX-110 spectrometer. Purity was verified via thin layer chromatography (TLC) on precoated silica gel G-25-UV254 plates with ethyl acetateand $\mathrm{n}$-hexane solvent systems.

\section{Synthesis of ethyl 4-chlorobenzoate (2)}

4-Chlorobenzoic acid $(1 ; 5.0 \mathrm{~g})$ was refluxed in $20.0 \mathrm{~mL}$ absolute ethanol and $2.0 \mathrm{~mL}$ concentrated $\mathrm{H}_{2} \mathrm{SO}_{4}$ in a $250 \mathrm{~mL}$ round bottom (RB) flask for $3 \mathrm{~h}$. After completion of the reaction which was verified by TLC, reaction mixture was taken up in a separating funnel followed by the addition of $200 \mathrm{~mL}$ distilled water and concentrated aqueous $\mathrm{Na}_{2} \mathrm{CO}_{3}$ solution $(\mathrm{pH}$ = 8-10). $50 \mathrm{~mL}$ diethylether was employed to extract the ester from the aqueous layer which was obtained on evaporation.

\section{Synthesis of 4-chlorobenzohydrazide (3)}

Ethyl 4-chlorobenzoate (2; $0.03 \mathrm{~mol})$ was stirred with $2.0 \mathrm{~mL} 80 \%$ hydrazine hydrate in $15.0 \mathrm{~mL}$ methanol in a $100 \mathrm{~mL}$ RB flask for $2 \mathrm{~h}$. After final TLC, the product was precipitated out by the addition of excess cold distilled water, separated by filtration and washed with $\mathrm{n}$-hexane.

\section{Synthesis of 5-(4-chlorophenyl)-1,3,4- oxadiazol-2-thiol (4)}

The mixture of 4-chlorobenzohydrazide (3; 0.03 $\mathrm{mol}$ ) and $50.0 \mathrm{~mL}$ absolute ethanol in a $250 \mathrm{~mL}$ $\mathrm{RB}$ flask was basified by $\mathrm{KOH}(0.03 \mathrm{~mol})$ on reflux. Carbon disulfide $(0.06 \mathrm{~mol})$ was added at room temperature. The mixture was refluxed for $5 \mathrm{~h}$ till single spot on TLC. Excess cold distilled water was poured into the reaction flask and acidified by dilute $\mathrm{HCl}(\mathrm{pH}$ of 2-3). The precipitates were filtered and washed with distilled water.

\section{Synthesis of ethyl 2-(5-(4-chlorophenyl)-1,3,4- oxadiazol-2-ylthio)acetate (5)}

The compound $4(0.03 \mathrm{~mol})$ was dissolved in 13 $\mathrm{mL}$ DMF in a $50 \mathrm{~mL}$ RB flask. $\mathrm{NaH}(0.12 \mathrm{~g})$ was added and stirred for $30 \mathrm{~min}$. Ethyl 2bromoacetate $(3.3 \mathrm{~mL})$ was added and further stirred for $3.5 \mathrm{~h}$. On single spot through TLC, excess ice cold distilled water was poured to flask for precipitation. The precipitates were isolated through filtration, washed and dried.

Synthesis of 2-(5-(4-chlorophenyl)-1,3,4oxadiazol-2-ylthio)acetohydrazide (6)

The ester $5(0.03 \mathrm{~mol})$ in $20.0 \mathrm{~mL}$ methanol was stirred with $2.0 \mathrm{~mL} 80 \%$ hydrazine hydrate strictly at room temperature in a $100 \mathrm{~mL}$ RB flask for $2.5 \mathrm{~h}$. After complete reaction, the addition of excess cold distilled water resulted in precipitation. The product was separated by filtration and washed off by $n$-hexane.

\section{Synthesis of N'-substituted-2-(5-(4- chlorophenyl)-1,3,4-oxadiazol-2- ylthio)acetohydrazide (8a-p)}

The compound $6(0.001 \mathrm{~mol})$ was taken in $17 \mathrm{~mL}$ methanol in a $50 \mathrm{~mL}$ RB flask followed by the addition of arylaldehydes (7a-p; $0.001 \mathrm{~mol})$. The reaction mixture was stirred for $2 \mathrm{~h}$. After complete reaction, the distilled water was added and the resulting precipitates were filtered, washed with distilled water and dried to afford the title compounds.

\section{Evaluation of antibacterial activity}

The antibacterial activity assay was executed as reported in the literature [10,11]. Antibacterial activity was performed in sterile 96-wells microplates under aseptic conditions. The variation in absorbance is noted which proportional to the microbial cell number is increasing in a log phase of growth.

\section{Statistical analysis}

All computations were executed in triplicate and statistical analysis was performed with Microsoft Excel 2010. The results are expressed as mean $\pm \operatorname{SEM}(n=3)$. Minimum inhibitory concentration (MIC) was computed with suitable dilutions (5 $30 \mu \mathrm{g} /$ well) for each sample and results calculated using EZ-Fit software (Perrella Scientific Inc, Amherst, USA).

\section{RESULTS}

The target molecules, N'-substituted-2-(5-(4chlorophenyl)-1,3,4-oxadiazol-2-

ylthio)acetohydrazide (8a-p) were synthesized as shown in Scheme-1. The synthesized compounds were further evaluated for the antibacterial activity. 


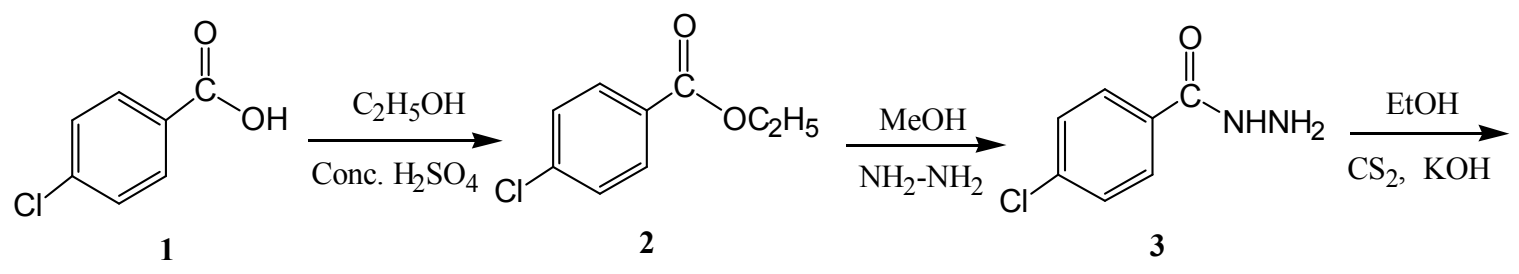<smiles>COC(=O)COC(=O)CSc1nnc(-c2ccc(Cl)cc2)o1</smiles>
4

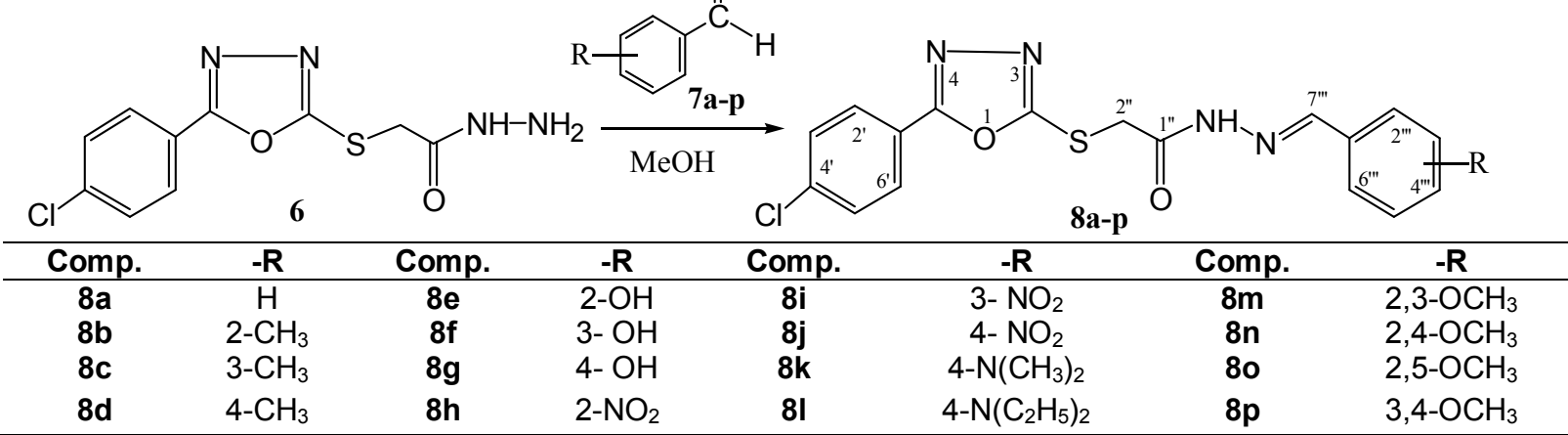

Scheme-1: Outline of synthesis of N'-substituted-2-(5-(4-chlorophenyl)-1,3,4-oxadiazol-2-ylthio)acetohydrazide $(8 a-p)$

Spectral characterization of synthesized molecules (2-6, 8a-p)

\section{Ethyl 4-chlorobenzoate (2)}

Yellow liquid; Yield: $84 \%$; Mol. formula: $\mathrm{C}_{9} \mathrm{H}_{9} \mathrm{ClO}_{2}$; Mol. mass: 184; IR $\left(\mathrm{KBr}, v_{\max } / \mathrm{cm}^{-1}\right)$ : $3105,1735,1599,697 ;{ }^{1} \mathrm{H}-\mathrm{NMR}(600 \mathrm{MHz}$,

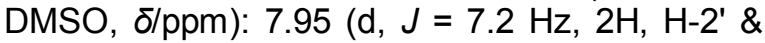
H-6'), 7.63 (d, J = 8.4 Hz, 2H, H-3' \& H-5'), 4.05 (q, $J=7.2 \mathrm{~Hz}, 2 \mathrm{H},-\mathrm{OCH}_{2} \mathrm{CH}_{3}$ ), 1.10 (t, $J=7.2$ $\left.\mathrm{Hz}, 3 \mathrm{H},-\mathrm{OCH}_{2} \mathrm{CH}_{3}\right)$; EIMS $(\mathrm{m} / \mathrm{z}): 186[\mathrm{M}+2]^{+}$, $184[\mathrm{M}]^{+}, 139\left[\mathrm{C}_{7} \mathrm{H}_{4} \mathrm{ClO}\right]^{+}, 111\left[\mathrm{C}_{6} \mathrm{H}_{4} \mathrm{Cl}\right]^{+}$.

\section{4-Chlorobenzohydrazide (3)}

White amorphous solid; Yield: 78 \%; M.P.: 162$164{ }^{\circ} \mathrm{C}$; Mol. formula: $\mathrm{C}_{7} \mathrm{H}_{7} \mathrm{ClN}_{2} \mathrm{O}$; Mol. mass: 170; IR ( $\left.\mathrm{KBr}, v_{\max } / \mathrm{cm}^{-1}\right): 3328,3118,1657,1609$, 703; ${ }^{1} \mathrm{H}-\mathrm{NMR}$ (600 MHz, DMSO, $\left.\delta / \mathrm{ppm}\right): 9.37$ (s, $1 \mathrm{H}, \mathrm{CONH}), 8.76(\mathrm{~s}, 2 \mathrm{H}, \mathrm{N}-\mathrm{H}), 7.89(\mathrm{~d}, J=7.8$ $\left.\mathrm{Hz}, 2 \mathrm{H}, \mathrm{H}-2^{\prime} \& \mathrm{H}-6^{\prime}\right), 7.59$ (d, J = 7.8 Hz, 2H, H-3' \& H-5'); EIMS $(\mathrm{m} / \mathrm{z}): 172[\mathrm{M}+2]^{+}, 170[\mathrm{M}]^{+}, 139$ $\left[\mathrm{C}_{7} \mathrm{H}_{4} \mathrm{ClO}\right]^{+}, 111\left[\mathrm{C}_{6} \mathrm{H}_{4} \mathrm{Cl}\right]^{+}$.

\section{5-(4-Chlorophenyl)-1,3,4-oxadiazol-2-thiol (4)}

White amorphous solid; Yield: 86 \%; M.P.: 170$172{ }^{\circ} \mathrm{C}$; Mol. formula: $\mathrm{C}_{8} \mathrm{H}_{5} \mathrm{CIN}_{2} \mathrm{OS}$; Mol. mass: 212; IR (KBr, $\left.v_{\text {max }} / \mathrm{cm}^{-1}\right): 3123,1665,1595,707$,
615; ${ }^{1} \mathrm{H}-\mathrm{NMR}(600 \mathrm{MHz}, \mathrm{DMSO}, \mathrm{\delta} / \mathrm{ppm}): 7.88$ (d, $\left.J=7.2 \mathrm{~Hz}, 2 \mathrm{H}, \mathrm{H}-2{ }^{\prime} \& \mathrm{H}-6^{\prime}\right), 7.63$ (d, $J=8.4 \mathrm{~Hz}$, $2 \mathrm{H}, \mathrm{H}-3^{\prime}$ \& $\left.\mathrm{H}-5^{\prime}\right)$; EIMS (m/z): $214[\mathrm{M}+2]^{+}, 212$ $[\mathrm{M}]^{+}, 139\left[\mathrm{C}_{7} \mathrm{H}_{4} \mathrm{ClO}\right]^{+}, \quad 137\left[\mathrm{C}_{7} \mathrm{H}_{4} \mathrm{ClN}\right]^{+}, 111$ $\left[\mathrm{C}_{6} \mathrm{H}_{4} \mathrm{Cl}\right]^{+}$.

\section{Ethyl 2-(5-(4-chlorophenyl)-1,3,4-oxadiazol-2- ylthio)acetate (5)}

White amorphous solid; Yield: 81 \%; M.P.: 174$176{ }^{\circ} \mathrm{C}$; Mol. formula: $\mathrm{C}_{12} \mathrm{H}_{11} \mathrm{ClN}_{2} \mathrm{O}_{3} \mathrm{~S}$; Mol. mass: 298; IR $\left(\mathrm{KBr}, v_{\max } / \mathrm{cm}^{-1}\right): 3145,1741,1679,1604$, 1058, 710; ${ }^{1} \mathrm{H}-\mathrm{NMR}(600 \mathrm{MHz}, \mathrm{DMSO}, \delta / \mathrm{ppm})$ : 7.96 (d, $\left.J=7.8 \mathrm{~Hz}, 2 \mathrm{H}, \mathrm{H}-2^{\prime} \& \mathrm{H}-6^{\prime}\right), 7.69$ (d, $J=$ $9.0 \mathrm{~Hz}, 2 \mathrm{H}, \mathrm{H}-3^{\prime}$ \& H-5'), 4.64 (s, 2H, H-2"), 3.93 (q, $J=7.2 \mathrm{~Hz}, 2 \mathrm{H},-\mathrm{OCH}_{2} \mathrm{CH}_{3}$ ), $1.03(\mathrm{t}, J=7.2$ $\left.\mathrm{Hz}, 3 \mathrm{H},-\mathrm{OCH}_{2} \mathrm{CH}_{3}\right) ; \operatorname{EIMS}(\mathrm{m} / \mathrm{z}): 300[\mathrm{M}+2]^{+}$, $298[\mathrm{M}]^{+}, 225\left[\mathrm{C}_{9} \mathrm{H}_{6} \mathrm{ClN}_{2} \mathrm{OS}\right]^{+}, 179\left[\mathrm{C}_{8} \mathrm{H}_{4} \mathrm{ClN}_{2} \mathrm{O}\right]^{+}$, $139\left[\mathrm{C}_{7} \mathrm{H}_{4} \mathrm{ClO}\right]^{+}, 137\left[\mathrm{C}_{7} \mathrm{H}_{4} \mathrm{ClN}\right]^{+}, 111\left[\mathrm{C}_{6} \mathrm{H}_{4} \mathrm{Cl}\right]^{+}$.

\section{2-(5-(4-Chlorophenyl)-1,3,4-oxadiazol-2- ylthio)acetohydrazide (6)}

White amorphous solid; Yield: 87 \%; M.P.: 178$180{ }^{\circ} \mathrm{C}$; Mol. formula: $\mathrm{C}_{10} \mathrm{H}_{9} \mathrm{CIN}_{4} \mathrm{O}_{2} \mathrm{~S}$; Mol. mass: 284; IR $\left(\mathrm{KBr}, v_{\max } / \mathrm{cm}^{-1}\right)$ : 3378, 3085, 1661, 1681 , 1601, 1065, 703; ${ }^{1} \mathrm{H}-\mathrm{NMR}(600 \mathrm{MHz}, \mathrm{DMSO}$, $\delta / \mathrm{ppm}): 9.41$ (s, 1H, CONH), $8.84(\mathrm{~s}, 2 \mathrm{H}, \mathrm{N}-\mathrm{H})$, $7.97\left(\mathrm{~d}, J=7.8 \mathrm{~Hz}, 2 \mathrm{H}, \mathrm{H}-2^{\prime} \& \mathrm{H}-6^{\prime}\right), 7.67$ (d, $J=$ $\left.9.0 \mathrm{~Hz}, 2 \mathrm{H}, \mathrm{H}-3^{\prime} \& \mathrm{H}-5^{\prime}\right), 4.61$ (s, $\left.2 \mathrm{H}, \mathrm{H}-2^{\prime \prime}\right)$; EIMS 
$(\mathrm{m} / \mathrm{z}): 286[\mathrm{M}+2]^{+}, 284[\mathrm{M}]^{+}, 225\left[\mathrm{C}_{9} \mathrm{H}_{6} \mathrm{CIN}_{2} \mathrm{OS}\right]^{+}$, $179 \quad\left[\mathrm{C}_{8} \mathrm{H}_{4} \mathrm{ClN}_{2} \mathrm{O}\right]^{+}, \quad 139 \quad\left[\mathrm{C}_{7} \mathrm{H}_{4} \mathrm{ClO}\right]^{+}, \quad 137$ $\left[\mathrm{C}_{7} \mathrm{H}_{4} \mathrm{ClN}\right]^{+}, 111\left[\mathrm{C}_{6} \mathrm{H}_{4} \mathrm{Cl}\right]^{+}$.

\section{$N^{\prime}$-Benzylidene-2-(5-(4-chlorophenyl)-1,3,4- oxadiazol-2-ylthio)acetohydrazide (8a)}

White amorphous solid; Yield: 73 \%; M.P.: 160$162{ }^{\circ} \mathrm{C}$; Mol. formula: $\mathrm{C}_{17} \mathrm{H}_{13} \mathrm{CIN}_{4} \mathrm{O}_{2} \mathrm{~S}$; Mol. mass: 372; IR (KBr, $\left.v_{\max } / \mathrm{cm}^{-1}\right): 3048,1659,1609,1071$, 690; ${ }^{1} \mathrm{H}-\mathrm{NMR}$ (600 MHz, DMSO, $\left.\delta / \mathrm{ppm}\right): 11.79$ (s, 1H, CONH), $8.22\left(\mathrm{~s}, 1 \mathrm{H}, \mathrm{H}-7^{\prime \prime \prime}\right), 7.97$ (d, $J=$ $\left.8.4 \mathrm{~Hz}, 2 \mathrm{H}, \mathrm{H}-2^{\prime} \& \mathrm{H}-6^{\prime}\right), 7.70$ (dd, $J=7.2,1.8$ $\mathrm{Hz}, 2 \mathrm{H}, \mathrm{H}-2$ "' \& H-6"'), 7.65 (d, J = 9.0 Hz, 2H, H3' \& H-5'), 7.47-7.43 (m, 3H, H-3'" to H-5"'), 4.67 (s, 2H, H-2"); EIMS (m/z): $374[\mathrm{M}+2]^{+}, 372[\mathrm{M}]^{+}$, $225\left[\mathrm{C}_{9} \mathrm{H}_{6} \mathrm{ClN}_{2} \mathrm{OS}\right]^{+}, 179 \quad\left[\mathrm{C}_{8} \mathrm{H}_{4} \mathrm{ClN}_{2} \mathrm{O}\right]^{+}, 147$ $\left[\mathrm{C}_{8} \mathrm{H}_{7} \mathrm{~N}_{2} \mathrm{O}\right]^{+}, 139\left[\mathrm{C}_{7} \mathrm{H}_{4} \mathrm{ClO}^{+}, 137\left[\mathrm{C}_{7} \mathrm{H}_{4} \mathrm{ClN}\right]^{+}, 111\right.$ $\left[\mathrm{C}_{6} \mathrm{H}_{4} \mathrm{Cl}\right]^{+}$.

$N^{\prime}$-(2-Methylbenzylidene)-2-(5-(4chlorophenyl)-1,3,4-oxadiazol-2ylthio)acetohydrazide (8b)

White amorphous solid; Yield: 79 \%; M.P.: 166$168{ }^{\circ} \mathrm{C}$; Mol. formula: $\mathrm{C}_{18} \mathrm{H}_{15} \mathrm{CIN}_{4} \mathrm{O}_{2} \mathrm{~S}$; Mol. mass: 386; IR $\left(\mathrm{KBr}, v_{\max } / \mathrm{cm}^{-1}\right): 3063,1651,1615,1074$, 699; ${ }^{1} \mathrm{H}-\mathrm{NMR}$ (600 MHz, DMSO, $\left.\delta / \mathrm{ppm}\right): 11.72$ (s, 1H, CONH), 8.31 (s, 1H, H-7"'), 7.97 (d, $J=$ $8.4 \mathrm{~Hz}, 2 \mathrm{H}, \mathrm{H}-2^{\prime}$ \& $\left.\mathrm{H}-6^{\prime}\right), 7.75$ (dd, $J=9.0,1.8$ $\left.\mathrm{Hz}, 1 \mathrm{H}, \mathrm{H}-6{ }^{\prime \prime \prime}\right), 7.65$ (d, J = 9.0 Hz, 2H, H-3' \& H5'), 7.35-7.30 (m, 2H, H-4"' \& H-5'"'), 7.26 (d, J = $\left.7.2 \mathrm{~Hz}, 1 \mathrm{H}, \mathrm{H}-3^{\prime \prime \prime}\right), 4.67$ (s, 2H, H-2"), 2.44 (s, $\left.3 \mathrm{H}, \mathrm{CH}_{3}-2^{\prime \prime \prime}\right)$; EIMS (m/z): $388[\mathrm{M}+2]^{+}, 386[\mathrm{M}]^{+}$, $225\left[\mathrm{C}_{9} \mathrm{H}_{6} \mathrm{CIN}_{2} \mathrm{OS}\right]^{+}, \quad 179 \quad\left[\mathrm{C}_{8} \mathrm{H}_{4} \mathrm{ClN}_{2} \mathrm{O}\right]^{+}, 161$ $\left[\mathrm{C}_{9} \mathrm{H}_{9} \mathrm{~N}_{2} \mathrm{O}\right]^{+}, 139\left[\mathrm{C}_{7} \mathrm{H}_{4} \mathrm{ClO}\right]^{+}, 137\left[\mathrm{C}_{7} \mathrm{H}_{4} \mathrm{ClN}\right]^{+}, 111$ $\left[\mathrm{C}_{6} \mathrm{H}_{4} \mathrm{Cl}\right]^{+}$.

$N^{\prime}$-(3-Methylbenzylidene)-2-(5-(4chlorophenyl)-1,3,4-oxadiazol-2ylthio)acetohydrazide (8c)

White amorphous solid; Yield: 80 \%; M.P.: 162$164^{\circ} \mathrm{C}$; Mol. formula: $\mathrm{C}_{18} \mathrm{H}_{15} \mathrm{CIN}_{4} \mathrm{O}_{2} \mathrm{~S}$; Mol. mass: 386; IR $\left(\mathrm{KBr}, v_{\max } / \mathrm{cm}^{-1}\right)$ : 3056, 1676, 1602, 1047 , 696; ${ }^{1} \mathrm{H}-\mathrm{NMR}$ (600 MHz, DMSO, $\left.\delta / \mathrm{ppm}\right): 11.75$ (s, 1H, CONH), $8.16\left(\mathrm{~s}, 1 \mathrm{H}, \mathrm{H}-7^{\prime \prime \prime}\right), 7.95(\mathrm{~d}, J=$ $\left.9.0 \mathrm{~Hz}, 2 \mathrm{H}, \mathrm{H}-2^{\prime} \& \mathrm{H}^{\prime} 6^{\prime}\right), 7.63(\mathrm{~d}, J=8.4 \mathrm{~Hz}, 2 \mathrm{H}$, H-3' \& H-5'), 7.48 (d, J = 7.2 Hz, 1H, H-6"'), 7.32 (t, $\left.J=7.8 \mathrm{~Hz}, 1 \mathrm{H}, \mathrm{H}-5^{\prime \prime \prime}\right), 7.26\left(\mathrm{~s}, 1 \mathrm{H}, \mathrm{H}-2^{\prime \prime \prime}\right), 7.23$ (d, J = 7.2 Hz, 1H, H-4"'), 4.66 (s, 2H, H-2"), 2.33 (s, 3H, $\left.\mathrm{CH}_{3}-3^{\prime \prime \prime}\right)$; $\operatorname{EIMS~}(\mathrm{m} / \mathrm{z}): 388[\mathrm{M}+2]^{+}, 386$ $[\mathrm{M}]^{+}, 225\left[\mathrm{C}_{9} \mathrm{H}_{6} \mathrm{ClN}_{2} \mathrm{OS}\right]^{+}, 179\left[\mathrm{C}_{8} \mathrm{H}_{4} \mathrm{ClN}_{2} \mathrm{O}\right]^{+}, 161$ $\left[\mathrm{C}_{9} \mathrm{H}_{9} \mathrm{~N}_{2} \mathrm{O}\right]^{+}, 139\left[\mathrm{C}_{7} \mathrm{H}_{4} \mathrm{ClO}\right]^{+}, 137\left[\mathrm{C}_{7} \mathrm{H}_{4} \mathrm{ClN}\right]^{+}, 111$ $\left[\mathrm{C}_{6} \mathrm{H}_{4} \mathrm{Cl}\right]^{+}$.
$N$ '-(4-Methylbenzylidene)-2-(5-(4chlorophenyl)-1,3,4-oxadiazol-2ylthio)acetohydrazide (8d)

White amorphous solid; Yield: 78 \%; M.P.: 170$172{ }^{\circ} \mathrm{C}$; Mol. formula: $\mathrm{C}_{18} \mathrm{H}_{15} \mathrm{CIN}_{4} \mathrm{O}_{2} \mathrm{~S}$; Mol. mass: 386; IR (KBr, $\left.v_{\text {max }} / \mathrm{cm}^{-1}\right)$ : 3046, 1665, 1619, 1056 , 692; ${ }^{1} \mathrm{H}-\mathrm{NMR}$ (600 MHz, DMSO, $\left.\delta / \mathrm{ppm}\right): 11.72$ (s, 1H, CONH), 8.18 (s, 1H, H-7"'), 7.96 (d, $J=$ $\left.8.4 \mathrm{~Hz}, 2 \mathrm{H}, \mathrm{H}-2^{\prime} \& \mathrm{H}-6^{\prime}\right), 7.64(\mathrm{~d}, J=8.4 \mathrm{~Hz}, 2 \mathrm{H}$, $\left.\mathrm{H}-3^{\prime} \& \mathrm{H}-5^{\prime}\right), 7.58\left(\mathrm{~d}, J=7.8 \mathrm{~Hz}, 2 \mathrm{H}, \mathrm{H}-2^{\prime \prime \prime} \& \mathrm{H}-\right.$ 6"'), 7.24 (d, $J=7.8 \mathrm{~Hz}, 2 \mathrm{H}, \mathrm{H}-3$ "' \& H-5'"'), 4.65 (s, 2H, H-2"), 2.34 (s, 3H, $\mathrm{CH}_{3}-4 "$ "'); EIMS ( $\mathrm{m} / \mathrm{z}$ ): $388[\mathrm{M}+2]^{+}, 386[\mathrm{M}]^{+}, 225\left[\mathrm{C}_{9} \mathrm{H}_{6} \mathrm{CIN}_{2} \mathrm{OS}\right]^{+}, 179$ $\left[\mathrm{C}_{8} \mathrm{H}_{4} \mathrm{ClN}_{2} \mathrm{O}\right]^{+}, 161\left[\mathrm{C}_{9} \mathrm{H}_{9} \mathrm{~N}_{2} \mathrm{O}\right]^{+}, 139\left[\mathrm{C}_{7} \mathrm{H}_{4} \mathrm{ClO}^{+}\right.$, $137\left[\mathrm{C}_{7} \mathrm{H}_{4} \mathrm{ClN}\right]^{+}, 111\left[\mathrm{C}_{6} \mathrm{H}_{4} \mathrm{Cl}\right]^{+}$.

N'-(2-Hydroxybenzylidene)-2-(5-(4chlorophenyl)-1,3,4-oxadiazol-2ylthio)acetohydrazide (8e)

Dirty white amorphous solid; Yield: 87 \%; M.P.: 212-214 ${ }^{\circ} \mathrm{C}$; Mol. formula: $\mathrm{C}_{17} \mathrm{H}_{13} \mathrm{CIN}_{4} \mathrm{O}_{3} \mathrm{~S}$; Mol. mass: 388 ; IR $\left(\mathrm{KBr}, v_{\max } / \mathrm{cm}^{-1}\right): 3064,1678$, 1611, 1061, 688; ${ }^{1} \mathrm{H}-\mathrm{NMR}$ (600 MHz, DMSO, $\delta / p p m): 11.73$ (s, 1H, CONH), 8.35 (s, 1H, H-7"'), 7.97 (d, $\left.J=9.0 \mathrm{~Hz}, 2 \mathrm{H}, \mathrm{H}-2^{\prime} \& \mathrm{H}^{-} 6^{\prime}\right), 7.71$ (dd, $J$ $\left.=7.8,1.8 \mathrm{~Hz}, 1 \mathrm{H}, \mathrm{H}-6^{\prime \prime \prime}\right), 7.65(\mathrm{~d}, J=8.4 \mathrm{~Hz}, 2 \mathrm{H}$, H-3' \& H-5'), 7.56 (dd, J = 7.2, $\left.1.2 \mathrm{~Hz}, 1 \mathrm{H}, \mathrm{H}-3^{\prime \prime \prime}\right)$, 7.25 (ddd, $\left.J=7.2,1.8 \mathrm{~Hz}, 1 \mathrm{H}, \mathrm{H}-4{ }^{\prime \prime \prime}\right), 6.85$ (t, $J=$ $\left.7.2 \mathrm{~Hz}, 1 \mathrm{H}, \mathrm{H}-5{ }^{\prime \prime \prime}\right), 4.66$ (s, 2H, H-2"); EIMS $(\mathrm{m} / \mathrm{z}): 390[\mathrm{M}+2]^{+}, 388[\mathrm{M}]^{+}, 225\left[\mathrm{C}_{9} \mathrm{H}_{6} \mathrm{ClN}_{2} \mathrm{OS}\right]^{+}$, $179 \quad\left[\mathrm{C}_{8} \mathrm{H}_{4} \mathrm{ClN}_{2} \mathrm{O}\right]^{+}, \quad 163 \quad\left[\mathrm{C}_{8} \mathrm{H}_{7} \mathrm{~N}_{2} \mathrm{O}_{2}\right]^{+}, 139$ $\left[\mathrm{C}_{7} \mathrm{H}_{4} \mathrm{ClO}\right]^{+}, 137\left[\mathrm{C}_{7} \mathrm{H}_{4} \mathrm{ClN}\right]^{+}, 111\left[\mathrm{C}_{6} \mathrm{H}_{4} \mathrm{Cl}\right]^{+}$.

\section{N'-(3-Hydroxybenzylidene)-2-(5-(4-} chlorophenyl)-1,3,4-oxadiazol-2ylthio)acetohydrazide (8f)

White amorphous solid; Yield: 82 \%; M.P.: 218$220{ }^{\circ} \mathrm{C}$; Mol. formula: $\mathrm{C}_{17} \mathrm{H}_{13} \mathrm{CIN}_{4} \mathrm{O}_{3} \mathrm{~S}$; Mol. mass: 388; IR (KBr, $\left.v_{\text {max }} / \mathrm{cm}^{-1}\right)$ : 3035, 1679, 1612, 1072, 698; ${ }^{1} \mathrm{H}-\mathrm{NMR}$ (600 MHz, DMSO, $\left./ \mathrm{ppm}\right): 11.74$ (s, 1H, CONH), $9.64\left(\mathrm{~s}, 1 \mathrm{H}, \mathrm{HO}-3^{\prime \prime \prime}\right), 8.12(\mathrm{~s}, 1 \mathrm{H}$, H-7'"'), 7.97 (d, $\left.J=7.8 \mathrm{~Hz}, 2 \mathrm{H}, \mathrm{H}-2^{\prime} \& \mathrm{H}-6^{\prime}\right), 7.65$ (d, $\left.J=9.0 \mathrm{~Hz}, 2 \mathrm{H}, \mathrm{H}-3^{\prime} \& \mathrm{H}-5^{\prime}\right), 7.24$ (t, $J=7.8$ $\left.\mathrm{Hz}, 1 \mathrm{H}, \mathrm{H}-5^{\prime \prime \prime}\right), 7.15$ (s, 1H, H-2'"'), 7.09 (d, $J=$ $\left.7.8 \mathrm{~Hz}, 1 \mathrm{H}, \mathrm{H}-6^{\prime \prime \prime}\right), 6.83$ (dd, $J=7.8,2.4 \mathrm{~Hz}, 1 \mathrm{H}$, H-4"'), 4.66 (s, 2H, H-2"); EIMS ( $\mathrm{m} / \mathrm{z}): 390$ $[\mathrm{M}+2]^{+}, \quad 388[\mathrm{M}]^{+}, \quad 225 \quad\left[\mathrm{C}_{9} \mathrm{H}_{6} \mathrm{ClN}_{2} \mathrm{OS}\right]^{+}, \quad 179$ $\left[\mathrm{C}_{8} \mathrm{H}_{4} \mathrm{ClN}_{2} \mathrm{O}\right]^{+}, 163\left[\mathrm{C}_{8} \mathrm{H}_{7} \mathrm{~N}_{2} \mathrm{O}_{2}\right]^{+}, 139\left[\mathrm{C}_{7} \mathrm{H}_{4} \mathrm{ClO}^{+}\right.$, $137\left[\mathrm{C}_{7} \mathrm{H}_{4} \mathrm{ClN}\right]^{+}, 111\left[\mathrm{C}_{6} \mathrm{H}_{4} \mathrm{Cl}\right]^{+}$.

N'-(4-Hydroxybenzylidene)-2-(5-(4chlorophenyl)-1,3,4-oxadiazol-2ylthio)acetohydrazide (8g)

White amorphous solid; Yield: 77 \%; M.P.: 234$236{ }^{\circ} \mathrm{C}$; Mol. formula: $\mathrm{C}_{17} \mathrm{H}_{13} \mathrm{ClN}_{4} \mathrm{O}_{3} \mathrm{~S}$; Mol. mass: 
388; IR ( $\left.\mathrm{KBr}, v_{\max } / \mathrm{cm}^{-1}\right): 3039,1674,1601,1078$, 704; ${ }^{1} \mathrm{H}-\mathrm{NMR}$ (600 MHz, DMSO, $\left.\delta / \mathrm{ppm}\right): 11.74$ (s, 1H, CONH), 8.09 (s, 1H, H-7"'), 7.96 (d, J = $8.4 \mathrm{~Hz}, 2 \mathrm{H}, \mathrm{H}-2$ ' \& H-6'), 7.64 (d, J = 8.4 Hz, $2 \mathrm{H}$, H-3' \& H-5'), 7.52 (d, J = 9.0 Hz, 2H, H-2"' \& H6"'), 6.80 (d, J = 9.0 Hz, 2H, H-3"' \& H-5"'), 4.62 (s, 2H, H-2"); EIMS (m/z): $390[\mathrm{M}+2]^{+}, 388[\mathrm{M}]^{+}$, $225\left[\mathrm{C}_{9} \mathrm{H}_{6} \mathrm{CIN}_{2} \mathrm{OS}\right]^{+}, 179 \quad\left[\mathrm{C}_{8} \mathrm{H}_{4} \mathrm{CIN}_{2} \mathrm{O}\right]^{+}, 163$ $\left[\mathrm{C}_{8} \mathrm{H}_{7} \mathrm{~N}_{2} \mathrm{O}_{2}\right]^{+}, 139\left[\mathrm{C}_{7} \mathrm{H}_{4} \mathrm{ClO}\right]^{+}, 137\left[\mathrm{C}_{7} \mathrm{H}_{4} \mathrm{ClN}\right]^{+}$, $111\left[\mathrm{C}_{6} \mathrm{H}_{4} \mathrm{Cl}\right]^{+}$.

N'-(2-Nitrobenzylidene)-2-(5-(4-chlorophenyl)1,3,4-oxadiazol-2-ylthio)acetohydrazide (8h)

Yellow amorphous solid; Yield: 73 \%; M.P.: 212$214{ }^{\circ} \mathrm{C}$; Mol. formula: $\mathrm{C}_{17} \mathrm{H}_{12} \mathrm{CIN}_{5} \mathrm{O}_{4} \mathrm{~S}$; Mol. mass: 417; IR ( $\left.\mathrm{KBr}, v_{\text {max }} / \mathrm{cm}^{-1}\right)$ : 3084, 1673, 1613, 1066 , 701; ${ }^{1} \mathrm{H}-\mathrm{NMR}(600 \mathrm{MHz}, \mathrm{DMSO}, \delta / \mathrm{ppm}): 12.09$ (s, 1H, CONH), 8.33 (s, 1H, H-7"'), 8.30 (d, J = $9.0 \mathrm{~Hz}, 1 \mathrm{H}, \mathrm{H}-6$ '"'), 8.26 (d, J = 9.0 Hz, 2H, H-2' \& H-6'), 7.98 (dd, J = 9.0, 2.4 Hz, 1H, H-3"'), 7.977.95 (m, 2H, H-4"' \& H-5"'), 7.65 (d, $J=8.4 \mathrm{~Hz}$, 2H, H-3' \& H-5'), 4.70 (s, 2H, H-2"); EIMS ( $\mathrm{m} / \mathrm{z}$ ): $419[\mathrm{M}+2]^{+}, 417[\mathrm{M}]^{+}, 225\left[\mathrm{C}_{9} \mathrm{H}_{6} \mathrm{CIN}_{2} \mathrm{OS}\right]^{+}, 192$ $\left[\mathrm{C}_{8} \mathrm{H}_{6} \mathrm{~N}_{3} \mathrm{O}_{3}\right]^{+}, 179\left[\mathrm{C}_{8} \mathrm{H}_{4} \mathrm{ClN}_{2} \mathrm{O}\right]^{+}, 139\left[\mathrm{C}_{7} \mathrm{H}_{4} \mathrm{ClO}\right]^{+}$, $137\left[\mathrm{C}_{7} \mathrm{H}_{4} \mathrm{ClN}\right]^{+}, 111\left[\mathrm{C}_{6} \mathrm{H}_{4} \mathrm{Cl}\right]^{+}$.

N'-(3-Nitrobenzylidene)-2-(5-(4-chlorophenyl)1,3,4-oxadiazol-2-ylthio)acetohydrazide (8i)

White amorphous solid; Yield: 76 \%; M.P.: 222$224{ }^{\circ} \mathrm{C}$; Mol. formula: $\mathrm{C}_{17} \mathrm{H}_{12} \mathrm{CIN}_{5} \mathrm{O}_{4} \mathrm{~S}$; Mol. mass: 417; IR ( $\left.\mathrm{KBr}, v_{\max } / \mathrm{cm}^{-1}\right): 3075,1651,1606,1057$, 693; ${ }^{1} \mathrm{H}-\mathrm{NMR}$ (600 MHz, DMSO, $\left.\delta / p p m\right): 12.05$ (s, 1H, CONH), $8.52(\mathrm{t}, J=1.8 \mathrm{~Hz}, 1 \mathrm{H}, \mathrm{H}-2$ '"), 8.36 (s, 1H, H-7"'), 8.26 (dd, $J=9.0,1.8 \mathrm{~Hz}, 1 \mathrm{H}$, H-6"'), 8.16 (d, J = 7.8 Hz, 1H, H-4"'), 7.96 (d, J = $\left.9.0 \mathrm{~Hz}, 2 \mathrm{H}, \mathrm{H}-\mathrm{2}^{\prime} \& \mathrm{H}-6^{\prime}\right), 7.74(\mathrm{t}, J=8.4 \mathrm{~Hz}, 1 \mathrm{H}$, H-5'"), 7.65 (d, J = 8.4 Hz, 2H, H-3' \& H-5'), 4.72 (s, 2H, H-2"); EIMS (m/z): $419[\mathrm{M}+2]^{+}, 417[\mathrm{M}]^{+}$, $225\left[\mathrm{C}_{9} \mathrm{H}_{6} \mathrm{CIN}_{2} \mathrm{OS}\right]^{+}, \quad 192 \quad\left[\mathrm{C}_{8} \mathrm{H}_{6} \mathrm{~N}_{3} \mathrm{O}_{3}\right]^{+}, 179$ $\left[\mathrm{C}_{8} \mathrm{H}_{4} \mathrm{CIN}_{2} \mathrm{O}\right]^{+}, 139\left[\mathrm{C}_{7} \mathrm{H}_{4} \mathrm{ClO}\right]^{+}, 137\left[\mathrm{C}_{7} \mathrm{H}_{4} \mathrm{CIN}\right]^{+}$, $111\left[\mathrm{C}_{6} \mathrm{H}_{4} \mathrm{Cl}\right]^{+}$.

N'-(4-Nitrobenzylidene)-2-(5-(4-chlorophenyl)1,3,4-oxadiazol-2-ylthio)acetohydrazide (8j)

Yellow amorphous solid; Yield: 75 \%; M.P.: 240$242{ }^{\circ} \mathrm{C}$; Mol. formula: $\mathrm{C}_{17} \mathrm{H}_{12} \mathrm{CIN}_{5} \mathrm{O}_{4} \mathrm{~S}$; Mol. mass: 417; IR ( $\left.\mathrm{KBr}, v_{\max } / \mathrm{cm}^{-1}\right): 3081,1659,1619,1069$, 691; ${ }^{1} \mathrm{H}-\mathrm{NMR}$ (600 MHz, DMSO, $\delta / \mathrm{ppm}$ ): 12.08 (s, 1H, CONH), 8.43 (s, 1H, H-7'"'), 8.09 (d, $J=$ $7.8 \mathrm{~Hz}, 2 \mathrm{H}, \mathrm{H}-3^{\prime \prime \prime} \& \mathrm{H}-5$ "'), 8.05 (d, $J=9.6 \mathrm{~Hz}$, $2 \mathrm{H}, \mathrm{H}-2$ "' \& H-6"'), 7.96 (d, J = $7.8 \mathrm{~Hz}, 2 \mathrm{H}, \mathrm{H}-2$ ' \& H-6'), 7.66 (d, J = 9.0 Hz, 2H, H-3' \& H-5'), 4.67 (s, 2H, H-2"); EIMS (m/z): $419[\mathrm{M}+2]^{+}, 417[\mathrm{M}]^{+}$, $225\left[\mathrm{C}_{9} \mathrm{H}_{6} \mathrm{CIN}_{2} \mathrm{OS}\right]^{+}, \quad 192 \quad\left[\mathrm{C}_{8} \mathrm{H}_{6} \mathrm{~N}_{3} \mathrm{O}_{3}\right]^{+}, 179$
$\left[\mathrm{C}_{8} \mathrm{H}_{4} \mathrm{ClN}_{2} \mathrm{O}\right]^{+}, 139\left[\mathrm{C}_{7} \mathrm{H}_{4} \mathrm{ClO}\right]^{+}, 137\left[\mathrm{C}_{7} \mathrm{H}_{4} \mathrm{ClN}\right]^{+}$, $111\left[\mathrm{C}_{6} \mathrm{H}_{4} \mathrm{Cl}\right]^{+}$.

N'-(4-(Dimethylamino)benzylidene)-2-(5-(4chlorophenyl)-1,3,4-oxadiazol-2ylthio)acetohydrazide (8k)

Yellow amorphous solid; Yield: $71 \%$; M.P.: 170$172{ }^{\circ} \mathrm{C}$; Mol. formula: $\mathrm{C}_{19} \mathrm{H}_{18} \mathrm{CIN}_{5} \mathrm{O}_{2} \mathrm{~S}$; Mol. mass: 415; IR $\left(\mathrm{KBr}, v_{\max } / \mathrm{cm}^{-1}\right): 3043,1679,1617,1064$, 701; ${ }^{1} \mathrm{H}-\mathrm{NMR}$ (600 MHz, DMSO, $\left.\delta / \mathrm{ppm}\right): 11.48$ (s, 1H, CONH), 8.04 (s, 1H, H-7'"), 7.96 (d, $J=$ $\left.9.0 \mathrm{~Hz}, 2 \mathrm{H}, \mathrm{H}-22^{\prime} \& \mathrm{H}^{-6}\right), 7.63$ (d, J = 8.4 Hz, 2H, $\left.\mathrm{H}-3^{\prime} \& \mathrm{H}-5^{\prime}\right), 7.47$ (d, J = 8.4 Hz, 2H, H-2'" \& H6"'), 6.69 (d, J = 9.0 Hz, 2H, H-3"' \& H-5"'), 4.59 (s, 2H, H-2"), 2.96 (s, 6H, $\left(\mathrm{CH}_{3}\right)_{2} \mathrm{~N}-4$ "'); EIMS $(\mathrm{m} / \mathrm{z}): 417[\mathrm{M}+2]^{+}, 415[\mathrm{M}]^{+}, 225\left[\mathrm{C}_{9} \mathrm{H}_{6} \mathrm{CIN}_{2} \mathrm{OS}\right]^{+}$, $190 \quad\left[\mathrm{C}_{10} \mathrm{H}_{12} \mathrm{~N}_{3} \mathrm{O}\right]^{+}, \quad 179 \quad\left[\mathrm{C}_{8} \mathrm{H}_{4} \mathrm{CIN}_{2} \mathrm{O}\right]^{+}, 139$ $\left[\mathrm{C}_{7} \mathrm{H}_{4} \mathrm{ClO}\right]^{+}, 137\left[\mathrm{C}_{7} \mathrm{H}_{4} \mathrm{CIN}\right]^{+}, 111\left[\mathrm{C}_{6} \mathrm{H}_{4} \mathrm{Cl}\right]^{+}$.

\section{N'-(4-(Diethylamino)benzylidene)-2-(5-(4- chlorophenyl)-1,3,4-oxadiazol-2- ylthio)acetohydrazide (8I)}

Yellow amorphous solid; Yield: 79 \%; M.P.: 174$176{ }^{\circ} \mathrm{C}$; Mol. formula: $\mathrm{C}_{21} \mathrm{H}_{22} \mathrm{CIN}_{5} \mathrm{O}_{2} \mathrm{~S}$; Mol. mass: 443; IR (KBr, $\left.v_{\text {max }} / \mathrm{cm}^{-1}\right): 3058,1634,1610,1059$, 689; ${ }^{1} \mathrm{H}-\mathrm{NMR}$ (600 MHz, DMSO, $\left.\delta / \mathrm{ppm}\right): 11.45$ (s, 1H, CONH), 8.01 (s, 1H, H-7"'), 7.96 (d, $J=$ $9.0 \mathrm{~Hz}, 2 \mathrm{H}, \mathrm{H}-2^{\prime}$ \& H-6'), 7.63 (d, J = 8.4 Hz, 2H, H-3' \& H-5'), 7.44 (d, J = 8.4 Hz, 2H, H-2'" \& H6"'), 6.64 (d, J = 9.0 Hz, 2H, H-3"' \& H-5"'), 4.58 (s, 2H, H-2"), 2.60 (q, J $=7.2 \mathrm{~Hz}, 4 \mathrm{H}$, $\left.\left(\mathrm{CH}_{3} \mathrm{CH}_{2}\right)_{2} \mathrm{~N}-4 " '\right), 1.09(\mathrm{t}, \quad J=7.2 \mathrm{~Hz}, 6 \mathrm{H}$, $\left(\mathrm{CH}_{3} \mathrm{CH}_{2}\right)_{2} \mathrm{~N}-4$ "'); EIMS (m/z): $445[\mathrm{M}+2]^{+}, 443$ $\left[\mathrm{M}^{+}, 225\left[\mathrm{C}_{9} \mathrm{H}_{6} \mathrm{CIN}_{2} \mathrm{OS}\right]^{+}, 212\left[\mathrm{C}_{8} \mathrm{H}_{5} \mathrm{CIN}_{2} \mathrm{OS}\right]^{+}\right.$, $190 \quad\left[\mathrm{C}_{11} \mathrm{H}_{16} \mathrm{~N}_{3}\right]^{+}, \quad 179 \quad\left[\mathrm{C}_{8} \mathrm{H}_{4} \mathrm{CIN}_{2} \mathrm{O}\right]^{+}, 139$ $\left[\mathrm{C}_{7} \mathrm{H}_{4} \mathrm{ClO}\right]^{+}, 137\left[\mathrm{C}_{7} \mathrm{H}_{4} \mathrm{ClN}\right]^{+}, 111\left[\mathrm{C}_{6} \mathrm{H}_{4} \mathrm{Cl}\right]^{+}$.

\section{N'-(2,3-Dimethoxybenzylidene)-2-(5-(4- chlorophenyl)-1,3,4-oxadiazol-2- ylthio)acetohydrazide $(8 \mathrm{~m})$}

White amorphous solid; Yield: 83 \%; M.P.: 164$166{ }^{\circ} \mathrm{C}$; Mol. formula: $\mathrm{C}_{19} \mathrm{H}_{17} \mathrm{CIN}_{4} \mathrm{O}_{4} \mathrm{~S}$; Mol. mass: 432; IR ( $\left.\mathrm{KBr}, v_{\max } / \mathrm{cm}^{-1}\right)$ : 3067, 1633, 1602, 1055, 708; ${ }^{1} \mathrm{H}-\mathrm{NMR}$ (600 MHz, DMSO, $\left.\delta / \mathrm{ppm}\right): 11.73$ (s, 1H, CONH), 8.32 (s, 1H, H-7"'), 7.96 (d, $J=$ $9.0 \mathrm{~Hz}, 2 \mathrm{H}, \mathrm{H}-2$ ' \& H-6'), 7.64 (d, J = 9.0 Hz, 2H, H-3' \& H-5'), 7.55 (d, J=8.4 Hz, 1H, H-6'"'), 7.43 (dd, $\left.J=7.8,1.8 \mathrm{~Hz}, 1 \mathrm{H}, \mathrm{H}-4{ }^{\prime \prime \prime}\right), 7.11$ (t, $J=7.8$ $\mathrm{Hz}, 1 \mathrm{H}, \mathrm{H}-5$ "'), 4.65 (s, 2H, H-2"), 3.84 (s, 3H, $\left.\mathrm{CH}_{3} \mathrm{O}-3^{\prime \prime \prime}\right), 3.77$ (s, 3H, $\mathrm{CH}_{3} \mathrm{O}-2$ "'); $\operatorname{EIMS~(m/z):~}$ $434[\mathrm{M}+2]^{+}, 432[\mathrm{M}]^{+}, 225\left[\mathrm{C}_{9} \mathrm{H}_{6} \mathrm{CIN}_{2} \mathrm{OS}\right]^{+}, 179$ $\left[\mathrm{C}_{8} \mathrm{H}_{4} \mathrm{CIN}_{2} \mathrm{O}\right]^{+}, 139\left[\mathrm{C}_{7} \mathrm{H}_{4} \mathrm{ClO}\right]^{+}, 137\left[\mathrm{C}_{7} \mathrm{H}_{4} \mathrm{CIN}\right]^{+}$, $111\left[\mathrm{C}_{6} \mathrm{H}_{4} \mathrm{Cl}\right]^{+}$. 
$N^{\prime}$-(2,4-Dimethoxybenzylidene)-2-(5-(4chlorophenyl)-1,3,4-oxadiazol-2ylthio)acetohydrazide (8n)

White amorphous solid; Yield: 85 \%; M.P.: 166$168{ }^{\circ} \mathrm{C}$; Mol. formula: $\mathrm{C}_{19} \mathrm{H}_{17} \mathrm{CIN}_{4} \mathrm{O}_{4} \mathrm{~S}$; Mol. mass: 432; IR (KBr, $\left.v_{\text {max }} / \mathrm{cm}^{-1}\right): 3061,1643,1604,1058$, 699; ${ }^{1} \mathrm{H}-\mathrm{NMR}$ (600 MHz, DMSO, $\left.\delta / \mathrm{ppm}\right): 11.61$ (s, 1H, CONH), 8.27 (s, 1H, H-7"'), 7.96 (d, $J=$ $\left.9.0 \mathrm{~Hz}, 2 \mathrm{H}, \mathrm{H}-2^{\prime} \& \mathrm{H}-6^{\prime}\right), 7.73(\mathrm{~d}, J=8.4 \mathrm{~Hz}, 1 \mathrm{H}$, H-6'"), 7.65 (d, $\left.J=9.0 \mathrm{~Hz}, 2 \mathrm{H}, \mathrm{H}-3^{\prime} \& \mathrm{H}-5^{\prime}\right), 6.62$ $\left(\mathrm{d}, J=2.4 \mathrm{~Hz}, 1 \mathrm{H}, \mathrm{H}-3^{\prime \prime \prime}\right), 6.57$ (dd, $J=8.4,1.8$ $\left.\mathrm{Hz}, 1 \mathrm{H}, \mathrm{H}-5^{\prime \prime \prime}\right), 4.61$ (s, 2H, H-2"), 3.85 (s, 3H, $\left.\mathrm{CH}_{3} \mathrm{O}-2{ }^{\prime \prime \prime}\right), 3.82$ (s, 3H, $\left.\mathrm{CH}_{3} \mathrm{O}-4{ }^{\prime \prime \prime}\right) ; \operatorname{EIMS}(\mathrm{m} / \mathrm{z})$ : $434[\mathrm{M}+2]^{+}, 432[\mathrm{M}]^{+}, 225\left[\mathrm{C}_{9} \mathrm{H}_{6} \mathrm{ClN}_{2} \mathrm{OS}\right]^{+}, 179$ $\left[\mathrm{C}_{8} \mathrm{H}_{4} \mathrm{ClN}_{2} \mathrm{O}\right]^{+}, 139\left[\mathrm{C}_{7} \mathrm{H}_{4} \mathrm{ClO}\right]^{+}, 137\left[\mathrm{C}_{7} \mathrm{H}_{4} \mathrm{ClN}\right]^{+}$, $111\left[\mathrm{C}_{6} \mathrm{H}_{4} \mathrm{Cl}\right]^{+}$.

$N^{\prime}$-(2,5-Dimethoxybenzylidene)-2-(5-(4chlorophenyl)-1,3,4-oxadiazol-2ylthio)acetohydrazide (80)

Dirty white amorphous solid; Yield: 80 \%; M.P.: 174-176 ${ }^{\circ} \mathrm{C}$; Mol. formula: $\mathrm{C}_{19} \mathrm{H}_{17} \mathrm{CIN}_{4} \mathrm{O}_{4} \mathrm{~S}$; Mol. mass: 432; IR $\left(\mathrm{KBr}, v_{\max } / \mathrm{cm}^{-1}\right): 3085,1631$, 1608, 1053, 696; ${ }^{1} \mathrm{H}-\mathrm{NMR}$ (600 MHz, DMSO, $\delta / \mathrm{ppm}): 11.73$ (s, 1H, CONH), 8.33 (s, 1H, H-7"'), $7.96\left(\mathrm{~d}, J=8.4 \mathrm{~Hz}, 2 \mathrm{H}, \mathrm{H}-2^{\prime} \& \mathrm{H}-6^{\prime}\right), 7.64(\mathrm{~d}, J=$ $\left.9.0 \mathrm{~Hz}, 2 \mathrm{H}, \mathrm{H}-3^{\prime} \& \mathrm{H}-5^{\prime}\right), 7.36(\mathrm{~d}, J=3.0 \mathrm{~Hz}, 1 \mathrm{H}$, H-6"'), 7.05 (d, $\left.J=7.8 \mathrm{~Hz}, 1 \mathrm{H}, \mathrm{H}-3^{\prime \prime \prime}\right), 7.01$ (dd, $J$
= 9.0, 3.0 Hz, 1H, H-4"'), 4.66 (s, 2H, H-2"), 3.80 (s, 3H, $\mathrm{CH}_{3} \mathrm{O}-5$ "'), 3.75 (s, 3H, $\mathrm{CH}_{3} \mathrm{O}-2$ "'); EIMS $(\mathrm{m} / \mathrm{z}): 434[\mathrm{M}+2]^{+}, 432[\mathrm{M}]^{+}, 225\left[\mathrm{C}_{9} \mathrm{H}_{6} \mathrm{ClN}_{2} \mathrm{OS}\right]^{+}$, $179 \quad\left[\mathrm{C}_{8} \mathrm{H}_{4} \mathrm{ClN}_{2} \mathrm{O}\right]^{+}, \quad 139 \quad\left[\mathrm{C}_{7} \mathrm{H}_{4} \mathrm{ClO}^{+}, \quad 137\right.$ $\left[\mathrm{C}_{7} \mathrm{H}_{4} \mathrm{CIN}\right]^{+}, 111\left[\mathrm{C}_{6} \mathrm{H}_{4} \mathrm{Cl}\right]^{+}$.

$N^{\prime}$-(3,4-Dimethoxybenzylidene)-2-(5-(4chlorophenyl)-1,3,4-oxadiazol-2-

ylthio)acetohydrazide (8p)

White amorphous solid; Yield: 84 \%; M.P.: 162$164{ }^{\circ} \mathrm{C}$; Mol. formula: $\mathrm{C}_{19} \mathrm{H}_{17} \mathrm{CIN}_{4} \mathrm{O}_{4} \mathrm{~S}$; Mol. mass: 432; IR (KBr, $\left.v_{\text {max }} / \mathrm{cm}^{-1}\right)$ : 3078, 1689, 1605, 1059 , 711; ${ }^{1} \mathrm{H}$-NMR (600 MHz, DMSO, $\left.\delta / \mathrm{ppm}\right): 11.69$ (s, $1 \mathrm{H}, \mathrm{CONH}), 8.12\left(\mathrm{~s}, 1 \mathrm{H}, \mathrm{H}-7^{\prime \prime \prime}\right), 7.94(\mathrm{~d}, J=$ $\left.9.0 \mathrm{~Hz}, 2 \mathrm{H}, \mathrm{H}-2^{\prime} \& \mathrm{H}^{\prime} 6^{\prime}\right), 7.63(\mathrm{~d}, J=9.0 \mathrm{~Hz}, 2 \mathrm{H}$, H-3' \& H-5'), 7.31 (d, J = 1.8 Hz, 1H, H-2'"), 7.18 (dd, $\left.J=8.4,1.8 \mathrm{~Hz}, 1 \mathrm{H}, \mathrm{H}-6{ }^{\prime \prime}\right), 6.98$ (d, $J=8.4$ $\left.\mathrm{Hz}, 1 \mathrm{H}, \mathrm{H}-5^{\prime \prime \prime}\right), 4.64$ (s, 2H, H-2"), 3.80 (s, 3H, $\left.\mathrm{CH}_{3} \mathrm{O}-3^{\prime \prime \prime}\right), 3.79$ (s, $\left.3 \mathrm{H}, \mathrm{CH}_{3} \mathrm{O}-4{ }^{\prime \prime \prime}\right)$; EIMS $(\mathrm{m} / \mathrm{z})$ : $434[\mathrm{M}+2]^{+}, 432[\mathrm{M}]^{+}, 225\left[\mathrm{C}_{9} \mathrm{H}_{6} \mathrm{CIN}_{2} \mathrm{OS}\right]^{+}, 179$ $\left[\mathrm{C}_{8} \mathrm{H}_{4} \mathrm{ClN}_{2} \mathrm{O}\right]^{+}, 139\left[\mathrm{C}_{7} \mathrm{H}_{4} \mathrm{ClO}\right]^{+}, 137\left[\mathrm{C}_{7} \mathrm{H}_{4} \mathrm{ClN}\right]^{+}$, $111\left[\mathrm{C}_{6} \mathrm{H}_{4} \mathrm{Cl}\right]^{+}$.

\section{Antibacterial activity}

The results of antibacterial activity results are shown in Tables 1 and 2.

Table 1: Antibacterial activity (inhibition) of the synthesized compounds

\begin{tabular}{lccccc}
\hline \multirow{2}{*}{ Compound } & \multicolumn{5}{c}{ Inhibition (\%) } \\
\cline { 2 - 6 } & S. typhi (-) & E. coli (-) & P. aeroginosa (-) & B. subtilis (+) & S. aureus (+) \\
\hline $\mathbf{6}$ & $76.62 \pm 2.00$ & $81.16 \pm 1.79$ & $68.04 \pm 1.65$ & $64.23 \pm 3.06$ & $64.55 \pm 2.55$ \\
8a & $62.79 \pm 3.65$ & $58.53 \pm 1.42$ & $43.56 \pm 1.98$ & $51.95 \pm 1.75$ & $43.75 \pm 2.35$ \\
8b & $48.58 \pm 1.06$ & $43.53 \pm 2.53$ & $20.05 \pm 2.84$ & $38.18 \pm 2.23$ & $56.70 \pm 3.70$ \\
8c & $53.92 \pm 2.13$ & $49.32 \pm 2.58$ & $38.76 \pm 1.72$ & $46.59 \pm 1.77$ & $47.35 \pm 3.05$ \\
8d & $60.64 \pm 2.22$ & $57.63 \pm 2.14$ & $28.97 \pm 5.00$ & $53.23 \pm 2.41$ & $56.45 \pm 1.55$ \\
8e & $82.89 \pm 2.25$ & $79.84 \pm 2.43$ & $76.75 \pm 1.60$ & $78.77 \pm 2.41$ & $71.45 \pm 4.15$ \\
8f & $69.95 \pm 2.87$ & $72.16 \pm 1.36$ & $51.60 \pm 3.76$ & $58.05 \pm 1.79$ & $52.00 \pm 1.70$ \\
8g & $65.59 \pm 1.77$ & $53.21 \pm 2.16$ & $59.74 \pm 0.67$ & $54.18 \pm 1.06$ & $66.55 \pm 3.45$ \\
8h & $51.86 \pm 3.05$ & $50.63 \pm 2.77$ & $51.86 \pm 1.75$ & $51.09 \pm 1.94$ & $45.60 \pm 5.00$ \\
8i & $54.75 \pm 1.55$ & $51.16 \pm 4.36$ & $45.88 \pm 2.47$ & $53.64 \pm 2.59$ & $63.30 \pm 4.30$ \\
8j & $75.25 \pm 1.40$ & $74.05 \pm 2.49$ & $65.36 \pm 1.44$ & $66.55 \pm 3.76$ & $70.55 \pm 0.65$ \\
8k & $67.45 \pm 1.67$ & $61.74 \pm 1.64$ & $53.25 \pm 2.01$ & $60.86 \pm 2.95$ & $58.10 \pm 1.00$ \\
8l & $64.90 \pm 1.32$ & $71.11 \pm 2.23$ & $59.02 \pm 2.32$ & $57.50 \pm 1.18$ & $59.60 \pm 2.60$ \\
8m & $67.65 \pm 3.22$ & $64.95 \pm 1.33$ & $52.16 \pm 3.61$ & $53.09 \pm 2.55$ & $56.60 \pm 4.30$ \\
8n & $69.56 \pm 1.76$ & $69.16 \pm 2.09$ & $55.10 \pm 0.15$ & $60.05 \pm 1.34$ & $60.95 \pm 0.35$ \\
80 & $75.05 \pm 2.08$ & $67.74 \pm 2.11$ & $57.73 \pm 1.00$ & $60.59 \pm 3.14$ & $57.50 \pm 2.20$ \\
8p & $66.76 \pm 1.67$ & $60.63 \pm 1.88$ & $46.44 \pm 1.70$ & $52.14 \pm 3.22$ & $55.55 \pm 3.35$ \\
\hline Ciprofloxacin & $\mathbf{9 1 . 8 3 \pm 0 . 0 5}$ & $\mathbf{9 1 . 6 5 \pm 1 . 4 7}$ & $\mathbf{9 0 . 5 6 \pm 1 . 1 1}$ & $\mathbf{9 0 . 8 9 \pm 1 . 0 5}$ & $\mathbf{9 2 . 0 5 \pm 2 . 3 2}$ \\
\hline
\end{tabular}


Table 2: Antibacterial activity (MIC) of the synthesized compounds

\begin{tabular}{lccccc}
\hline \multirow{2}{*}{ Compound } & \multicolumn{5}{c}{ MIC $(\boldsymbol{\mu M})$} \\
\cline { 2 - 6 } & S. typhi $(-)$ & E. coli $(-)$ & P. aeroginosa (-) & B. subtilis (+) & S. aureus (+) \\
\hline $\mathbf{6}$ & $11.23 \pm 2.08$ & $13.52 \pm 2.40$ & $13.49 \pm 3.08$ & $13.35 \pm 5.00$ & $16.18 \pm 1.15$ \\
$\mathbf{8 a}$ & $10.37 \pm 2.13$ & $13.46 \pm 2.50$ & - & $17.54 \pm 1.50$ & - \\
$\mathbf{8 b}$ & - & - & - & - & $16.51 \pm 2.50$ \\
$\mathbf{8 c}$ & $18.21 \pm 4.83$ & - & - & - & $13.50 \pm 1.15$ \\
$\mathbf{8 d}$ & $14.29 \pm 3.67$ & $16.58 \pm 5.00$ & - & $11.94 \pm 5.00$ & $10.65 \pm 3.43$ \\
$\mathbf{8 e}$ & $12.03 \pm 4.92$ & $9.45 \pm 1.00$ & $10.53 \pm 2.97$ & $14.67 \pm 0.55$ & $19.09 \pm 1.38$ \\
$\mathbf{8 f}$ & $10.32 \pm 1.42$ & $11.21 \pm 1.60$ & $18.52 \pm 3.46$ & $15.44 \pm 5.00$ & $13.37 \pm 5.00$ \\
$\mathbf{8 g}$ & $12.93 \pm 3.00$ & $13.18 \pm 4.07$ & $15.47 \pm 1.23$ & $19.69 \pm 1.38$ & - \\
$\mathbf{8 h}$ & $19.08 \pm 5.00$ & $19.66 \pm 4.00$ & $19.02 \pm 4.08$ & $15.45 \pm 4.37$ & $12.13 \pm 5.00$ \\
$\mathbf{8 i}$ & $17.64 \pm 5.00$ & $16.32 \pm 1.20$ & - & $14.68 \pm 2.16$ & $10.98 \pm 2.64$ \\
$\mathbf{8 j}$ & $14.26 \pm 5.00$ & $12.03 \pm 4.13$ & $12.42 \pm 1.85$ & $14.40 \pm 3.81$ & $14.15 \pm 4.63$ \\
$\mathbf{8 k}$ & $14.15 \pm 0.42$ & $15.36 \pm 2.67$ & $18.13 \pm 1.12$ & $16.73 \pm 4.75$ & $16.03 \pm 3.38$ \\
$\mathbf{8 I}$ & $17.00 \pm 1.87$ & $14.02 \pm 5.00$ & $17.68 \pm 4.62$ & $14.41 \pm 2.31$ & $15.18 \pm 2.38$ \\
$\mathbf{8 m}$ & $11.41 \pm 1.25$ & $12.02 \pm 1.87$ & $18.44 \pm 3.11$ & $12.71 \pm 4.13$ & $14.31 \pm 3.00$ \\
$\mathbf{8 n}$ & $10.92 \pm 1.67$ & $12.76 \pm 1.33$ & $15.43 \pm 1.46$ & $11.01 \pm 3.94$ & $16.01 \pm 5.00$ \\
$\mathbf{8 0}$ & $12.10 \pm 1.00$ & $13.44 \pm 4.67$ & $14.91 \pm 3.00$ & $18.10 \pm 2.00$ & $17.90 \pm 4.00$ \\
$\mathbf{8 p}$ & $10.04 \pm 1.25$ & $11.90 \pm 4.20$ & - & $\mathbf{8 . 0 2} \pm \mathbf{0 . 3 3}$ & $\mathbf{8 . 4 1} \pm \mathbf{1 . 0 4}$ \\
\hline Ciprofloxacin & $\mathbf{9 . 1 3 \pm 2 . 0 0}$ & $\mathbf{8 . 9 0} \pm 1.65$ & $\mathbf{9 . 0 1 \pm 0 . 1 3}$ & & \\
\hline
\end{tabular}

\section{DISCUSSION}

The compound, $\mathbf{8 a}$, showed characteristic absorption bands of the IR spectra at 3048, $1659,1609,1071$ and 690. Its molecular formula, $\mathrm{C}_{17} \mathrm{H}_{13} \mathrm{CIN}_{4} \mathrm{O}_{2} \mathrm{~S}$ was shown by EIMS with [M] ${ }^{+}$ peak at $\mathrm{m} / \mathrm{z} 372$ and number of protons in ${ }^{1} \mathrm{H}$ NMR spectrum. The prominent fragmentation peaks of this molecule were depicted in Fig. 1. The three signals in ${ }^{1} \mathrm{H}$-NMR spectrum allotted to six protons of benzylidene moiety were $\delta 8.22$ $\left(1 \mathrm{H}, \mathrm{H}-7{ }^{\prime \prime \prime}\right), \delta 7.70\left(\mathrm{~J}=7.2,1.8 \mathrm{~Hz}, 2 \mathrm{H}, \mathrm{H}-2{ }^{\prime \prime \prime}\right.$ \& $\left.\mathrm{H}-6{ }^{\prime \prime \prime}\right)$ and $\delta 7.47-7.43\left(3 \mathrm{H}, \mathrm{H}-3\right.$ "' to $\left.\mathrm{H}-5{ }^{\prime \prime \prime}\right)$. Two doublets at $\delta 7.97$ with $J=8.4 \mathrm{~Hz}$ and at $\delta 7.65$ with $J=9.0 \mathrm{~Hz}$ were assigned to $\mathrm{H}-2{ }^{\prime}$ \& $\mathrm{H}-6^{\prime}$ and $\mathrm{H}-3$ ' \& $\mathrm{H}-5$ ', respectively. The signals resonating at $\delta 11.79(\mathrm{~s}, 1 \mathrm{H}, \mathrm{CONH})$ and $4.67(\mathrm{~s}, 2 \mathrm{H}, \mathrm{H}-2 ")$ confirmed the acetamidic protons.

On the basis of spectral analysis, the molecule 8a was named, N'-benzylidene-2-(5-(4chlorophenyl)-1,3,4-oxadiazol-2-

ylthio)acetohydrazide. The structures of other synthesized molecules were also corroborated likewise.

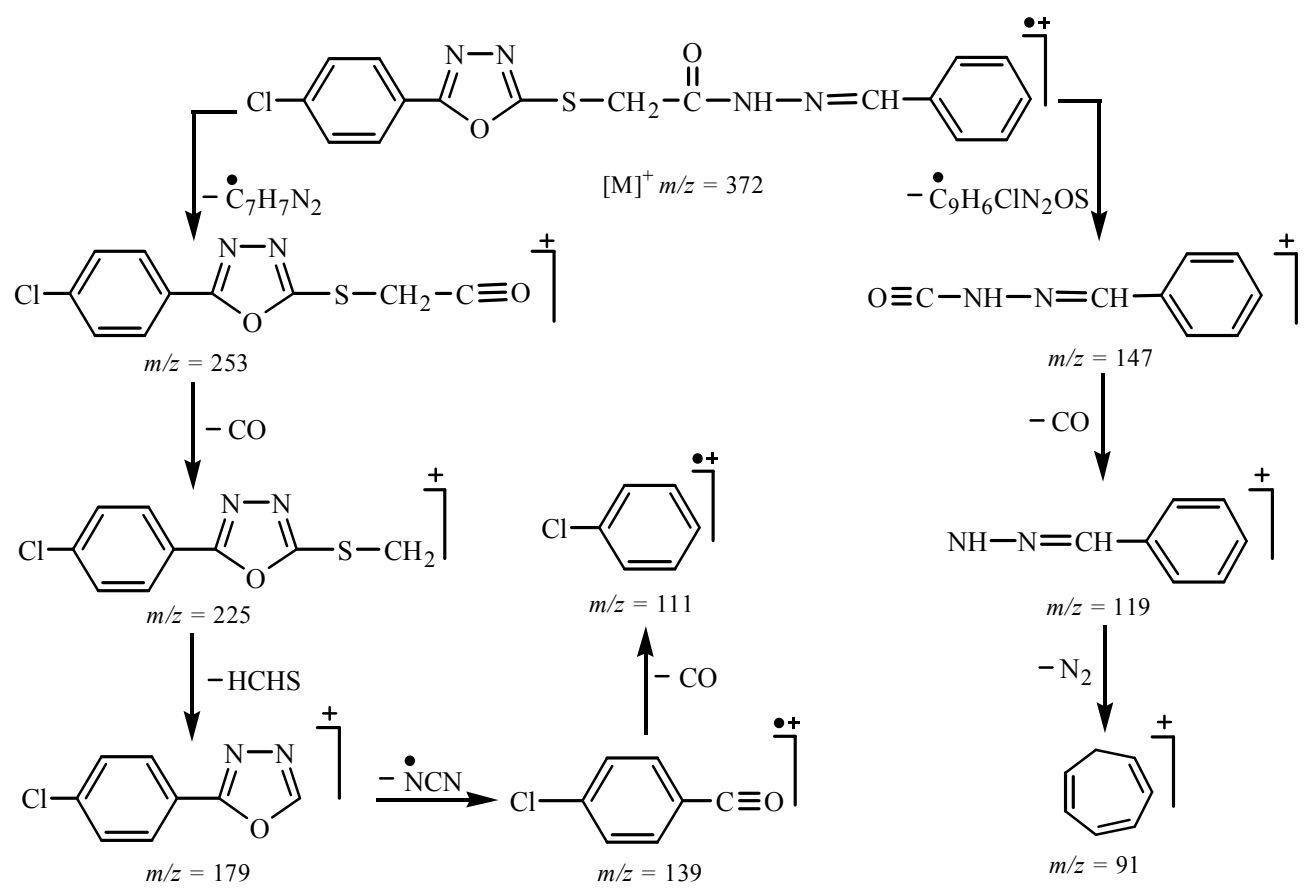

Figure 1: Mass fragmentation pattern of synthesized molecule $\mathbf{8 a}$ 
The activities of synthesized molecules were found to be higher against various bacterial strains of gram negative bacteria as compared to such type of other molecules [12-15]. The methylbenzylidenes (8b, $\mathbf{8 c} \quad \& \quad \mathbf{8 d})$ and nitrobenzylidene $(\mathbf{8} \mathbf{h}, \mathbf{8} \mathbf{i} \& \mathbf{8 j})$ showed the least activities except for $p$-substituted. Overall $\mathbf{8 a}, \mathbf{8 b}$, 8c, 8d, 8h, 8i and 8p executed a little low activity against some of bacterial strains. Among Gramnegative bacterial strains, $\mathbf{8 p}$ was the most active against $S$. typhi with MIC value of $10.04 \pm 1.25$ $\mu \mathrm{M}$ relative to the reference standard, ciprofloxacin with MIC value of $9.13 \pm 2.00 \mu \mathrm{M}$ and $8 \mathrm{e}$ against $E$. coli with MIC value of $9.45 \pm$ $1.00 \mu \mathrm{M}$ relative to $8.90 \pm 1.65 \mu \mathrm{M}$. The potent activities of $\mathbf{8 p}$ and $\mathbf{8 e}$ may be due to 3,4dimethoxy and 2-hydroxy benzylidenes respectively.

\section{CONCLUSION}

The relatively moderate MIC values indicate that the synthesized molecules possess potentials as antibacterial agents but there is need for further work to develop new drug candidates.

\section{REFERENCES}

1. Roy B, Chakraborty A, Ghosh SK, Basak A. Design, synthesis and bioactivity of catechin /epicatechin and 2-azetidinone derived chimeric molecules. Bioorg Med Chem Lett 2009; 19: 7007-7010.

2. Somani RR, Shirodkar PY. Oxadiazole, A biologically important heterocycle. Der Pharma Chemica 2009; 1(1): 130-140.

3. Jaiswal $N$, Singh $A K$, Singh $D$, Ahmad T. $A$ comprehensive review on antimicrobial activity of 1,3.4-Oxadiazole derivatives. Int Res J Pharm 2012; 3(3): 83-89

4. Khalid H, Aziz-ur-Rehman, Abbasi MA, Malik A, Rasool S, Nafeesa K, Ahmad I, Afzal S. Synthesis, spectral analysis and anti-bacterial study of $\mathrm{N}$-substituted derivatives of 2-(5-(1-(phenylsulfonyl)piperidin-4-yl)1,3,4-Oxadiazol-2-ylthio)acetamide. J Saudi Chem Soc 2013; doi: http://dx.doi.org/10.1016/j.jscs. 2013.05.001

5. Aziz-ur-Rehman, Fatima A, Abbasi MA, Rasool S, Malik A, Ashraf M, Ahmad I, Ejaz SA. Synthesis of new N-
(5-Chloro-2-methoxyphenyl)-4-(5-substituted-1,3,4Oxadiazol-2-ylthio)butanamide derivatives as suitable lipoxygenase inhibitors. J Saudi Chem Soc 2013; doi: http://dx.doi.org/10.1016/j.jscs.2013.02.006.

6. Hemavathi SN, Vishukumar BK, Lokanatha RKM. Synthesis and biological screening of some new 2,5disubstituted 1,3,4-Oxadiazoles. Int J Pharm Pharm Sci 2011; 3(4): 110-114.

7. Kumar S. Synthesis and biological activity of 5Substituted-2-amino-1,3,4-Oxadiazole derivatives. Turk J Chem 2011; 35: 99-108.

8. Aziz-ur-Rehman, Nafeesa K, Abbasi MA, Khalid H, Khan KM, Ashraf M, Ahmad I, Ejaz SA. Synthesis, spectral characterization and biological activity of $S$ substituted derivatives of 5-(4-Nitrophenyl)-1,3,4Oxadiazole-2-thiol. Asian J Pharm Hea Sci 2012; 2(3): 370-376.

9. Aziz-ur-Rehman, Siddiqui SZ, Abbas N, Abbasi MA, Khan KM, Shahid M, Mahmood Y, Akhtar NM, Lajis $\mathrm{NH}$. Synthesis, antibacterial screening and hemolytic activity of S-substituted derivatives of 5-Benzyl-1,3,4Oxadiazole-2-thiol. Int J Pharm Pharm Sci 2012; 4(2): 676-680.

10. Kaspady M, Narayanaswamy VK, Raju M, Rao GK. Synthesis, antibacterial activity of 2,4-disubstituted oxazoles and thiazoles as bioesters. Lett Drug Des Discov 2009; 6: 21-28.

11. Aziz-ur-Rehman, Nafeesa K, Abbasi MA, Kashfa $H$, Rasool S, Ahmad I, Arshad S. Synthesis, characterization and biological screening of various S-substituted derivatives of 5-(3-Nitrophenyl)-1,3,4Oxadiazole-2-thiol. Pak J Chem 2013; 3(2): 1-8.

12. Narsibhai $B D$, Mishra $D$, Vyavahare $L V$, Singh $A$. Thiazolidinone: Synthesis and biological studies. Arch Appl Sci Res 2012; 4(4): 1816-1820.

13. Rollas S, Küçükgüzel ŞG. Biological activities of hydrazone derivatives. Molecules 2007; 12: 19101939.

14. Shakya AK, El-Agbar Z. Synthesis and biological evaluation of Schiff bases of 2-Amino-5-(2chlorophenyl)-1,3,4-oxadiazole. Asian J Chem 2008; 20(4): 2515-2520.

15. Somani RR, Agrawal AG, Kalantri PP, Gavarkar PS, Clercq ED. Investigation of 1,3,4-Oxadiazole scaffold as potentially active compounds. Int J Drug Des Discov 2011; 2(1): 353-360. 